\title{
Stealing dimensions from metals
}

\author{
Although electrically charged black holes seem remote from superconductors and strange \\ metals in the laboratory, they might be intimately related by the holographic dualities discovered in \\ string theory.
}

\section{Jan Zaanen}

to $s$ it possible to force a three-dimensional (3D) zero-temperature quantum liquid to only flow in two dimensions by employing a static periodic potential in the third direction of non-infinite strength? Conventional quantum liquids submit to a no-go theorem. Both Fermi liquids and superconductors/superfluids eventually follow the principle of single particle quantum mechanics, in that their particle-like excitations will tunnel with a finite rate through the potential barrier. This is of more than merely theoretical interest, as the 'strange metals' forming the non-superconducting state in the cuprate superconductors, for example, are known to accomplish some form of this 'self-organized two-dimensionality'.

Writing in Nature Physics, Aristomenis Donos and Sean Hartnoll ${ }^{2}$ present a mathematical theory that describes strange metals for which losing a dimension is not unusual. The way this works is mind-boggling for the uninitiated: the investigation of this quantum matter is reduced to finding out how space-time quakes behave near the event horizon of an electrically charged black hole. The mathematical machinery at work is the anti-de Sitter/conformal field theory (AdS/CFT) correspondence, a powerful result of string theory, which has recently been mobilized to study electron systems in solids ${ }^{3}$. Remarkably, this mathematics finds serendipity on its side in this particular context: to simplify the gravitational computations Donos and Hartnoll used a unidirectional potential, to learn afterwards from condensed matter theorists that they had accidentally addressed the classic problem of dimensional reduction ${ }^{1,4}$.

Different from the reductionist outlook of string theory in the past, the AdS/ CFT correspondence ${ }^{3}$ can be viewed as a modern form of quantum statistical physics, aimed at the derivation of phenomenological theories that can describe highly collective, emergent properties of macroscopic matter. It is in essence a generalization of the 'weak-strong duality' of quantum field theory, relating a strongly interacting and severely fluctuating quantum stuff (the interest of condensed matter physics) to a weakly interacting dual world, that is now governed by Einstein's theory of general relativity in an AdS space-time with an extra dimension rooted in the holographic principle of quantum gravity.

Although the microscopic physics on the field theory side (large $N$ Yang-Mills theory) is completely detached from the interacting electrons of condensed matter, the states of matter that emerge on macroscopic scales are gravitationally encoded through generic solutions of the highly restrictive Einstein theory. Helped by the dialogue with condensed matter physicists, rapid advances were made recently and it is now established that all familiar states of matter have a gravitational dual: classical liquids correspond with Schwarzschild black holes, zero temperature Fermi-liquids and superconductors are described by 'stars' formed from charged fermions and Higgs fields, and so forth ${ }^{3}$.

However, AdS/CFT also works when conventional techniques fall short, specifically when dealing with the problem of strongly interacting fermions ${ }^{5}$. The simplest gravitational structures that describe finite density fermionic matter are charged black holes, and these dualize in entities that are entirely beyond the scope of established condensed matter theory. These 'holographic strange metals' ${ }^{6}$ have traits in common with a great riddle of condensed matter physics: the 'non Fermi-liquid' metals observed in high temperature $\left(T_{c}\right)$ superconductors and other strongly correlated electron systems ${ }^{5,7}$.

Identified shortly after the discovery of high $T_{\mathrm{c}}$ superconductivity in the late $1980 \mathrm{~s}$, these have since mystified the physics community. It is some form of highly collective 'quantum soup' exhibiting simple yet mysterious properties such as the 'local quantum criticality', referring to quantum fluctuations that are governed by a purely temporal scale invariance - this turns out to be precisely a trait of the holographic strange metal ${ }^{6}$. Another famous mystery is the unreasonable anisotropic nature of the electrical transport ${ }^{1}$. The copper oxides consist of stacks of copper oxide planes, and band structure calculations supported by optical data ${ }^{8}$ indicate that the system should be metallic in the direction perpendicular to the planes. Although the strange metal is conductive in planar directions, somehow coherent transport is completely suppressed in the perpendicular direction, showing an insulator-like electrical transport ${ }^{1,8}$.

A present focus of AdS/CFT research is incorporating the effects of a periodic ionic lattice into the holographical model, an important ingredient when dealing with electrons in solids. Breaking translational symmetry is not a favourite habit of general relativity and Donos and Hartnoll ${ }^{2}$ have to mobilize fancy technology to arrive at a flexible description of a corrugated black hole horizon, although they can only make this work for a unidirectional potential. For a small potential this describes a strange metal that is metallic in all directions, but when the potential exceeds a critical strength they find that an insulator forms exclusively in the direction of the potential - the 'planar' direction continues to be a perfect metal.

The key is that the localization mechanism in the strange metal is very different from either the wave interference behind band insulators or plain crystallization. The holographic metal-insulator transition is governed by a continuous renormalization flow, where the periodic potential turns from irrelevant to relevant, such that the energy-dependent conductivity decreases in the insulator in a power-law manner on lowering the energy, to disappear completely only at zero temperature and energy.

There is actually a precedent for such behaviour. This exploits the theoretical loophole that occurs in one space dimension, where it becomes possible to describe 
non-Fermi liquid 'quantum soups' - the Luttinger liquids. An array of such Luttinger liquids can be constructed, allowing the microscopic electrons to delocalize in two dimensions. But one can demonstrate ${ }^{4}$ that when the interactions inside the $1 \mathrm{D}$ metals become sufficiently strong the electron delocalization in the array of Luttinger liquids becomes irrelevant, yielding precisely the same continuous renormalization flow to a macroscopic metal 'losing a dimension' as is found in the holographic strange metal.
Although it remains to be seen whether the laboratory strange metals are literally such charged black hole 'holograms', AdS/CFT has at least the benefit of helping physicists to think differently, thereby exposing the spectacular beauty of these new forms of quantum matter.

Jan Zaanen is at the Instituut-Lorentz,

Universiteit Leiden, PO Box 9506, 2300 RA Leiden, The Netherlands.

e-mail: jan@lorentz.leidenuniv.nl
References

1. Anderson, P. W. The Theory of High $T_{c}$ Superconductivity in the High $T_{c}$ Superconductors (Princeton Univ. Press, 1997).

2. Donos, A. \& Hartnoll, S. A. Nature Phys. 9, 649-655 (2013)

3. Horowitz, G. T. (ed.) Black Holes in Higher Dimensions (Cambridge Univ. Press, 2012).

4. Emery, V. J. et al. Phys. Rev. Lett. 85, 2160-2163 (2000).

5. Zaanen, J. Science 319, 1205-1207 (2008).

6. Liu, H. Phys. Today 65, 68-69 (June 2012).

7. Zaanen, J. in 100 Years of Superconductivity (eds. Rogalla, H. \& Kes, P. H.) 92-117 (Chapman \& Hall, 2011).

8. Kim, J. H. et al. Physica C 247, 297-308 (1995).

Published online: 11 August 2013

\section{OXIDE HETEROSTRUCTURES}

\section{Hund rules with a twist}

The metallic sheet at the heterointerface between two different insulating and non-magnetic oxides displays seemingly conflicting ferromagnetic properties that may be explained by the presence of a spiral magnetic structure.

\section{Marc Gabay and Jean-Marc Triscone}

$\mathrm{S}$ cientific breakthroughs often occur thanks to a combination of intuition and serendipitous luck. Trivial as this statement may sound, it accurately qualifies the stunning discovery made almost ten years ago by A. Ohtomo and H. Y. Hwang ${ }^{1}$. The pair engineered a heterostructure composed of two strongly insulating oxide materials, lanthanum aluminate (LAO) and strontium titanate (STO), grown along the (001) orientation. Lo and behold, they observed a metallic-like region in the vicinity of the boundary layer between LAO and STO. Subsequently, it was established that a few-nanometre-thick conducting electron gas, a $2 \mathrm{DEG}$, may form on the STO side of the interface under specific growth conditions that include, for example, at least four unit cells of LAO grown on top of STO. Thus, increasing the thickness of one

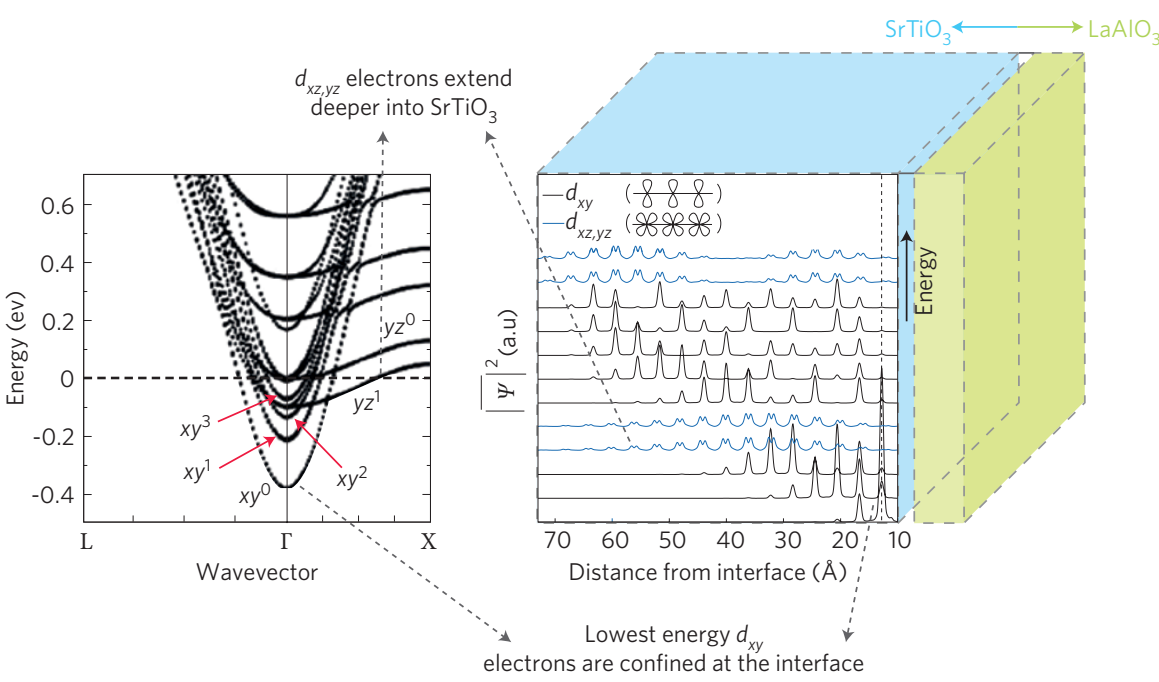

Figure 1 | Electronic structure. Left, The dispersion of the different subbands. The lowest energy subbands have a $d_{x y}$ character. Right, Extension of the wavefunctions $\Psi$ of the subbands through $\mathrm{SrTiO}_{3}$. The lowest energy $d_{x y}$ electrons are very spatially localized at the interface, whereas $d_{x z}$ and $d_{y z}$ extend further into the $\mathrm{SrTiO}_{3}$. Figure adapted with permission from: Left, ref. 15, (c) 2011 APS; Right, ref. 16, (c) 2009 APS.

part of the 'sandwich' triggers an insulator-metal transition.

Further experiments showcased several distinctive features of the LAO-STO 2DEG in comparison with electron gases in III-V semiconductors. Superconductivity was found at low temperature and the critical temperature could be controlled by means of a metallic gate. The spin and momentum of the charge carriers in the 2DEG are entangled, due to the so-called interfacial (Rashba) spin-orbit interaction - a signature of the breaking of inversion symmetry at the LAO-STO interface. The size of the Rashba energy rises by one order of magnitude on gate tuning and reaches the kinetic energy of the carriers, a rather unusual situation. These carriers originate from the $d$ electronic shell of the titanium (Ti) ions and come in two species, light $d_{x y}$ and heavier $d_{x z}$ and $d_{y z}$ electrons. The confinement of the electron gas at the interface deeply modifies the (bulk) STO electronic structure, and leads to a series of subbands (shown in Fig. 1). The $d_{x y}$ electrons occupy the lowest energy states of the 2DEG; they account for most of the charge in the system and a vast majority of those reside at - or in immediate proximity to - the interface. By contrast, $d_{x z}$ or $d_{y z}$ electrons naturally extend further away from the interface and amount to only a small fraction of the $d_{x y}$ population. Yet, their mobility reaches much higher values than that of the $d_{x y}$ states and they seem to play an important role in the electrical transport. 